\title{
Plant Diversity, Life Form and Phytochoria of Hamedan Alvand Region in Iran
}

\author{
Maryam Kolahi ${ }^{*}$, Morteza Atri² \\ ${ }^{1}$ Department of Biology, Faculty of Science, Shahid Chamran University of Ahvaz, Ahvaz, Iran \\ ${ }^{2}$ Department of Biology, Faculty of Science, Bu-Ali Sina University, Hamedan, Iran \\ Email: ${ }^{*}$ m.kolahi@scu.ac.ir
}

Received 2 August 2014; revised 26 September 2014; accepted 27 October 2014

Copyright (C) 2014 by authors and OALib.

This work is licensed under the Creative Commons Attribution International License (CC BY). http://creativecommons.org/licenses/by/4.0/

(c) (i) Open Access

\section{Abstract}

This study was carried out for determination and discrimination vegetation by Eco-phytosociology method. Flora of this area was determined by using available references. Based on collection we encountered about 290 specimens that belong to 167 genera and 41 families. The largest families in the area are Asteraceae (47 genera), Poaceae (34 genera), Lamiaceae (30 genera), Caryophylaceae (18 genera) Papilonaceae (17 genera). The floristic composition of the area is strongly influenced by large number of Irano-Turanian elements. The life form spectrum was characterized according to Raunkiers system. The life form spectrum observed was: Hemichryptophyte $(61.62 \%)$, Chamophyte $(15.14 \%)$, Therophyte $(14.79 \%)$, Phanerophyte $(4.58 \%)$ and Geophyte (3.87\%). The largest phytochoria distribution species is Irano-Turanian (59\%). Medicinal plants were identified that 71 medicinal plants species is belonging to 20 families and 57 genuses. Species riches as 290 plant species in this region distinguished by variable habitats, because of different ecologic factor and variation endogenous milieus. High variation of floristic showed variation biologic conditions and ecologic high power in this region for survivals. Anthropogenic impact of human and pasturing in this region are species downfall factors, especially rare species.

\section{Keywords}

Alvand, Flora, Life Form, Chorotype, Eco-Phytosociology Method, Iran

Subject Areas: Ecology, Plant Science

\section{Introduction}

Iran with an area of more than 1.6 million square $\mathrm{km}^{2}$ is the sixteenth largest country in the world, placed in the Middle East and surrounded by the Armenia, Azerbaijan and Caspian Sea, Turkmenistan on the north, Afghani-

*Corresponding author. 
stan and Pakistan on the east, Oman Sea and Persian Gulf on the south and Iraq and Turkey on the west. Iranian habitats support about 8000 species of flowering plants (belonging to 167 families and 1200 genera), of which almost 1700 are endemic [1]. These plant species grow on four Ecological Zones which have different physiographical and climatic conditions. These four ecological zones are: Hyrcanian, Zagross, Iran-o-Turanian, Plains Mountain, Khalij-o-Ommanian.

Iran is one of countries with diverse flora and fauna. This diversity results from its topography and diverse climatic conditions which led to the emergence of habitats that are suitable for the evolution and survival of various plant and animal species.

Clearance of natural vegetation to meet the demands of an ever increasing human population has been an ongoing process as long as permanent settlements existed. Ecological and environmental problems such as soil degradation, soil erosion and alteration of natural resources are just some of the negative effects resulting from the destruction of these habitats [2].

These habitats usually regenerate through natural processes after a significant disturbance of human activities such as cutting for fuel wood, construction material and clearance for cultivation [3]. Therefore, in order to maintain the ecological equilibrium and to meet the vegetation resource requirements of the population, scientific information on the composition, structure and distribution of species is the basis for habitat development.

\section{Study Area}

Alvand region is located in the west of Iran, and Hamedan, Asadabad and Touyserkan cities surround it. This region is located between western longitudes $48^{\circ} 10^{\prime}$ to $48^{\circ} 40^{\prime}$ and northern latitudes $34^{\circ} 30^{\prime}$ to $34^{\circ} 50^{\prime}$. The precipitation ranges between 206.1 and $420.7 \mathrm{~mm}$, respectively. Its maximal altitude is $3428 \mathrm{~m}$.

The special nature of this region and its historic sites attract tourists. The main sites of this region are the Ganjnameh, Takhteh-Nader, MeydanMishan, Kivarestan.

The climate of the study area is considered to be semi-arid, the annual precipitation being approximately 300 $\mathrm{mm}$. Rainfall occurs from October to May, with a maximum during November and February of each year. Another feature characterizing the precipitation in the study site is its irregular yearly distribution. The mean monthly temperatures vary between $1.91^{\circ} \mathrm{C}$ and $23.45^{\circ} \mathrm{C}$, the mean annual value being $10.88^{\circ} \mathrm{C}$. The annual potential evaporation far exceeds the annual rainfall with a mean annual amount of $1505 \mathrm{~mm}$, approximately estimated [4].

The aim of this study was to determine floristic composition and plant species diversity in the Hamedan Alvand region of Iran (Figure 1).

\section{Materials and Methods}

In this study the unit of study (endogenous milieu) in Eco-phytosociological method is used [5]. Endogenous milieu (special station) in Eco-phytosociological method is an area of vegetation that is homogenous view point of Floristic-Ecologic. In vegetations study, Endogenous milieu determine by physiognomic-floristic-ecological criteria. Establishment of releves (sampling unit in phytosociology) was carried out randomly in each Endogenous milieu (special station) for floristic-ecologic data collecting. In this order, all ecologic-floristic data were collected of each special station. Plant specimen deposited in the herbarium, of Bu-Ali Sina University in Hamadan, Iran. After providing herbarium labels were identified using available literature [6]-[12] and comparing with identical specimens in herbarium.

The chorology of each species was determined using published data [7]-[9] [13]. Determining the life form was done by Raunchiers classification [14] and then floristic list of this area provided in Table 1. The abbreviations used in the text and the floristic list is as follow: T: Therophyte, H: Hemicryptophyte, C: Chamaephyte, P: Phanerophyte, G: Geophyte, IT: Irano-Turanian, Z: Zagrosian, KO: Khaliji_Omani, M: Mediterranean, COS: Cosmopolite, H: Khazari.

\section{Results}

The result of study show that about 290 species belong to 167 genera and 41 families. Among the existing families, 34 families are Dicotyledonous, 5 families are monocotyledon, 1 family (Ephedraceae) is Gymnosperms and 1 family is Pteridophyta. Compositae with $17.2 \%$ species is the most abundant family in the area, followed by families of Poaceae, Labiatae with $11.4 \%, 11 \%$ species, respectively (Figure 2). In the paper checklist of all 


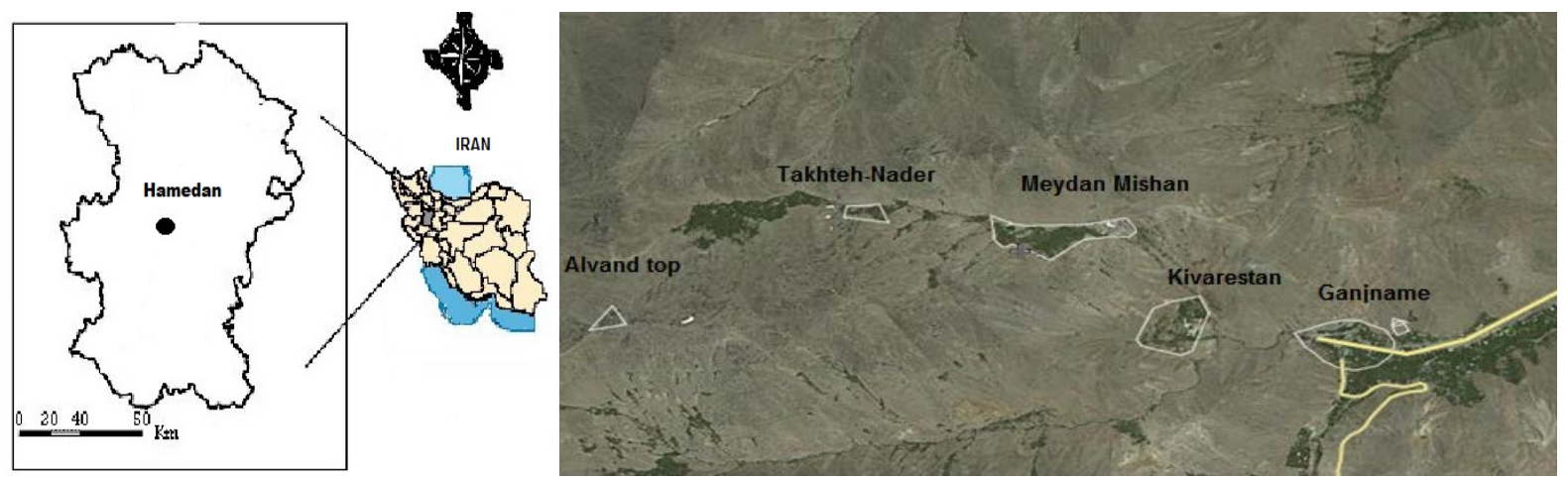

Figure 1. Topographic map of Hamedan Alvand region showing position of the area in Iran.

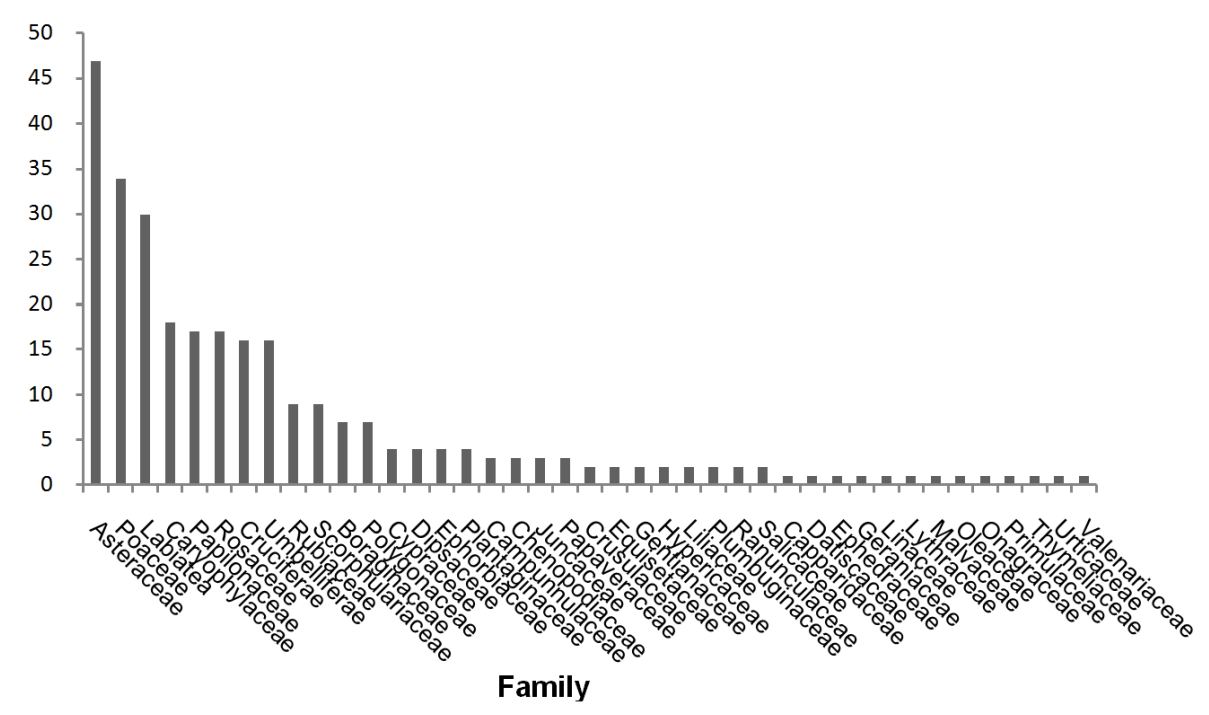

Figure 2. The pie chart of families Hamedan Alvand region.

species collected in Hamedan Alvand region is shown with information about their life forms and chorological types (Table 1). The jenera with the greatest number of species were Astragalus $2.7 \%$ species. The life-form spectrum was shown that a high proportion Hemichryptophyte (61.62\%) followed by Chamophyte (15.14\%), Therophyte (14.79\%), Phanerophyte (4.58\%) and Geophyte (3.87\%) (Figure 3). The chorology spectrum was shown: Hamedan Alvand region is geographically located main phytogeographic Irano-Turanian region. The most of plants chorotype with $58.62 \%$ is related to Irano-Toranian (Figure 4).

About 71 medicinal species were determined in this area that was shown in Table 1. Some of most important species are as follow: Echinops orientalis Trautv., Lactuca serriola L., Scariola orientalis (Boiss.) Sojak Alyssum lanigerum Dc. Euphorbia macroclada Bioss. Marrubium vulgare L. Mentha longifolia (L.) Hudson, Salvia multicaulis Vah1. Teucrium orientale L., Lotus corniculatus L., Plantago lanceolata L., Crataegus pontica C. Koch. Urticadioica L.

\section{Discussion}

The result of study show that the study area is very rich with refer to plant diversity. Among all plants Hemichryptophyte with $61.62 \%$ is dominant and Camophyte with $15.14 \%$ is in the next order. In facts life forms of the plants indicate the possibility of adaptation of plants to environmental factors especially climatic condition.

According to Archibold (1995) the frequency of Hemicryptophyte plants is due to cold and to altitude climate. The whole frequency of Hemicryptophyte among the plants of the region shows that the effect form of climate area is cold semi-arid to climatic of heights [15]. Therophyte adapted to the dryness of the region and shortage rainfall, because these plants spend vegetative period in the form of seed [16] [17]. 
Table 1. list of species, life form and phytochoria from Hamedan Alvand region.

\begin{tabular}{|c|c|c|c|}
\hline Scientific name & Life form & Phytocoria & Medicinal use \\
\hline \multicolumn{4}{|l|}{ ASTERACEAE } \\
\hline Achillea tenuifolia Lam. & $\mathrm{H}$ & IT & \\
\hline Aster alpinus L. & $\mathrm{H}$ & IT & \\
\hline Centaurea behen L. & $\mathrm{H}$ & IT & \\
\hline Centaurea iberica Trev. Ex. Spreng & $\mathrm{H}$ & H, IT, Z & \\
\hline Centaurea virgata Lam. & $\mathrm{H}$ & H, IT, Z & \\
\hline Cerastium dichotomum $\mathrm{L}$. & $\mathrm{H}$ & IT & \\
\hline Chondrilla juncea L. & $\mathrm{H}$ & IT & * \\
\hline Cichorium intybus L. & $\mathrm{H}$ & IT, H, KO & $*$ \\
\hline Cirsium congestum Fisch. \& Dc. & $\mathrm{H}$ & $\mathrm{Z}, \mathrm{IT}$ & \\
\hline Cirsium echinus Hand-Mzt. & $\mathrm{H}$ & IT, Z & \\
\hline Cirsium haussknechtii Boiss. & $\mathrm{H}$ & IT & \\
\hline Cirsium hygrophilum Boiss. & $\mathrm{H}$ & IT & \\
\hline Cirsium lappaceum M. B. & $\mathrm{H}$ & IT & \\
\hline Cirsium libanoticum Dc. & $\mathrm{H}$ & IT & \\
\hline Cousinia cylindracea Boiss. & $\mathrm{H}$ & IT, Z & \\
\hline Cousinia ecbatanensis Bornm. & $\mathrm{H}$ & IT & \\
\hline Cousinia elwendensis Bornm. & $\mathrm{H}$ & IT & \\
\hline Echinops macrophyllus Boiss. \& Hausskn. & $\mathrm{H}$ & IT & \\
\hline Echinops mosulensis Rechf. & $\mathrm{H}$ & IT & \\
\hline Echinops orientalis Trautv. & $\mathrm{H}$ & IT & $*$ \\
\hline Echinops ritrodes Bunge. & $\mathrm{H}$ & IT & \\
\hline Echinops tournefortii Trautv. & $\mathrm{H}$ & IT & \\
\hline Erigeron sp. & $\mathrm{T}$ & IT & \\
\hline Filago arvensis L. & $\mathrm{T}$ & IT & \\
\hline Filago pyramidata $\mathrm{L}$. & $\mathrm{T}$ & IT & \\
\hline Gundelia tournefortii L. & $\mathrm{H}$ & Z, IT, M, H & * \\
\hline Helichrysum oligocephalum Dc. & $\mathrm{H}$ & IT & \\
\hline Helichrysum psychrophilum Boiss. & $\mathrm{H}$ & IT & \\
\hline Inula britannica $\mathrm{L}$. & $\mathrm{H}$ & IT,Z & \\
\hline Inula helenium L. & $\mathrm{H}$ & IT & $*$ \\
\hline Lactuca serriola $\mathrm{L}$. & $\mathrm{H}$ & IT, Z, M, H & $*$ \\
\hline Micropus supinus L. & $\mathrm{T}$ & IT & $*$ \\
\hline Onopordon acanthium L. & $\mathrm{H}$ & IT, ES & \\
\hline Onopordon leptolepis Dc. & $\mathrm{H}$ & IT & \\
\hline Picnomon acarna (L.) Cass. & $\mathrm{T}$ & H, IT, Z & $*$ \\
\hline Picris strigosa M. B. & $\mathrm{H}$ & $\mathrm{Z}, \mathrm{IT}$ & \\
\hline Scariolao rientalis (Boiss.) Sojak & $\mathrm{H}$ & IT & * \\
\hline Scorzonera tortuosissima Boiss. & $\mathrm{H}$ & IT & \\
\hline Senecio vulgaris $\mathrm{L}$. & $\mathrm{T}$ & IT & * \\
\hline Serratula cerinthifolia Boiss. & $\mathrm{H}$ & IT, Z & \\
\hline Sonchus asper (L.) Hill & $\mathrm{H}$ & $\mathrm{I}, \mathrm{KO}, \mathrm{H}, \mathrm{M}$ & * \\
\hline Tanacetum parthenium Schult-Bip. & $\mathrm{C}$ & IT & $*$ \\
\hline Tanacetum persica & $\mathrm{H}$ & IT & \\
\hline Tanacetum polycephalum Schultz & $\mathrm{H}$ & IT & * \\
\hline Taraxacum syriacum Boiss. & $\mathrm{H}$ & IT, Z & \\
\hline Tragopogon graminifolius Dc. & $\mathrm{H}$ & H, IT & \\
\hline
\end{tabular}




\section{Continued}

Tripeluros pemumdisciforme Schultz

BORAGINACEAE

Alkanna orientalis (L.) Boiss.

Helotropium. sp.

Lappula. sp.

Myosotis olympica Boiss.

Onosma elwendicum Wettst.

Onosm amicrocarpum Dc.

Trichodesma aucheri Dc.

Trichodesma incanum (Bge.) A. Dc.

CAMPANULACEAE

Asyneuma multicaule Boiss

Asyneuma persicum (Dc.) Bronm.

Campanula latifolia $\mathrm{L}$.

CAPPARIDACEAE

Cleome iberica Dc.

CARYOPHYLLACEAE

Acanthophyllum microcephalum Boiss.

Arenaria insignis Litw.

Arenaria persica Boiss.

Arenaria serpyllifolia L.

Buffonia sp.

Cerastium inflatum Link ex. Desf.

Dianthus orientalis Adams

Dianthus tabrisianus BienertBoiss.

Gypsophila bicolor (Freyn \& Sint.) Grossh.

Gypsophila caricifolia Boiss.

Gypsophila pallida Stapf.

Herniaria glabra L.

Minuartia lineate Bornm.

Minuartia meyeri (Boiss.) Bornm.

Silene bupleuroides $\mathrm{L}$.

Silene chlorifolia Sm.

Silene noctiflora $\mathrm{L}$.

Stellaria media (L.) Cyr.

CHENOPODIACEAE

Chenopodium botrys L.

Kochia prostrata (L.) Schrad

Noaeamu cronata (Forsk.) Aschers Et. Schweinf

CRASSULACEAE

Rosularia elymaitica Berger

Rosularia sempervivum Berger

CRUCIFERAE

Alyssum dasycarpum Step. willd

Alyssum lanigerum Dc.

Alyssum marginatum Steud, Boiss.

Alyssum minus (L.) Rothm.

Alyssum szowitsianum Fisch. \& Mey.

Aubrietia parviflora Boiss.

$\mathrm{H}$

$\mathrm{H}$

$\mathrm{H}$

$\mathrm{H}$

$\mathrm{H}$

$\mathrm{H}$

$\mathrm{H}$

$\mathrm{H}$

$\mathrm{H}$

$\mathrm{H}$

C

H

$\mathrm{H}$

H

$\mathrm{H}$

C

$\mathrm{H}$

$\mathrm{H}$

$\mathrm{H}$

$\mathrm{H}$

$\mathrm{T}$

$\mathrm{H}$

C

$\mathrm{H}$

$\mathrm{T}$

$\mathrm{T}$

$\mathrm{H}$

H

$\mathrm{H}$

$\mathrm{H}$

$\mathrm{H}$

H

$\mathrm{T}$

$\mathrm{T}$

$\mathrm{T}$

$\mathrm{H}$
IT, Z

IT

IT

IT

IT

IT

IT

IT

IT

IT

IT

IT, M, KO

IT

IT

IT

IT

IT

IT, KO, Z

IT, KO

IT

IT, $\mathrm{KO}$

IT

IT

IT

IT

IT, KO, Z

IT

IT

IT

IT, M

IT, M

IT

IT, M, KO

IT

IT

IT

IT

IT

IT

IT

IT 


\section{Continued}

Barbarea plantaginea Dc.

Cardamine uliginosa M. B.

Carda ria draba (L.) Desv.

Clastopus vestitus (Desv.) Boiss.

Drabopsis verna C. Koch

Erysimum caespitosum Dc.

Fibigia suffruticosa (vent.) Sweet

Hesperis persica Boiss. (L.)

Nasturtium officinale R. Br.

Physoptychis gnaphalodes Boiss.

\section{CYPERACEAE}

Carex stenophylla Wahlenb

Cyperus difformis L.

Cyperus longus L.

Holoschoenus vulgaris

DATISCACEAE

Datisca cannabina L.

DIPSACACEAE

Cephalaria procera Fisch. \& Avel.

Pterocephalus canus Coult. Ex. Dc. Scabiosa argentea L.

Scabiosa flavida Boiss. \& Hausskn

EPHEDRACEAE

Ephedra major Host.

EQUISETACEAE

Equisetum arvense L.

Equisetum ramosissimum Desf.

EUPHORBIACEAE

Euphorbia boissieriana Prokh.

Euphorbia cheiradenia Boiss.\& Hohen.

Euphorbia decipiens Boiss. \& Buhse.

Euphorbia macroclada Bioss.

GENTIANACEAE

Centaurium minus (Moench)

Centaurium pulchellum Druce.

GERANIACEAE

Geranium montanum Habl. Ex.Pall

\section{JUNCACEAE}

Juncus articulatus L.

Juncus inflexus L.

Juncus rigidus Desf.

\section{HYPERICACEAE}

Hypericum perforatum L.

Hypericum scabrum L.

\section{LABIATAE}

Acinos graveolens (M. B.) Link

Marrubium astracanicum jacq.

Marrubium cuneatum Russell

$\mathrm{T}$

$\mathrm{H}$

$\mathrm{T}$

C

$\mathrm{T}$

$\mathrm{H}$

C

$\mathrm{T}$

C

$\mathrm{H}$

C

$\mathrm{H}$

G

$\mathrm{H}$

C

C

$\mathrm{H}$

$\mathrm{H}$

$\mathrm{H}$

C

G

G

$\mathrm{H}$

$\mathrm{H}$

$\mathrm{H}$

$\mathrm{H}$

$\mathrm{T}$

H

C

G

G

G

H

H

T

C

$\mathrm{H}$
IT

IT

IT, KO, Z

IT

IT

IT

IT, Z

IT

IT, KO, H

IT

IT

IT, KO, COS

IT, KO

IT

IT, KO

IT

IT

IT

IT

IT

IT

IT

IT

IT, KO, Z

IT

IT

IT

IT

IT

IT, Z

IT

IT, Z

IT

IT, M

IT, KO, Z, H

IT, KO

IT, KO 


\section{Continued}

\begin{tabular}{|c|c|c|c|}
\hline Marrubium vulgare $\mathrm{L}$. & $\mathrm{H}$ & IT, Z, KO, M & * \\
\hline Mentha longifolia (L.) Hudson. & $\mathrm{H}$ & IT, KO & * \\
\hline Nepeta crispa Willd & & IT & * \\
\hline Nepeta fissa C. A. Mey. & $\mathrm{H}$ & IT & \\
\hline Nepeta pungens (Bunge) Benth. & $\mathrm{T}$ & IT & \\
\hline Nepeta straussii Hausskn \& Bornm. & $\mathrm{T}$ & IT & * \\
\hline Phlomis anisodonta Boiss. & $\mathrm{H}$ & IT, KO, Z & \\
\hline Phlomis olivieri Benth. & $\mathrm{H}$ & IT, KO, Z & \\
\hline Phlomis persica Boiss. & $\mathrm{H}$ & IT, Z & \\
\hline Phlomis polioxantha Rechf. & $\mathrm{H}$ & IT, Z & \\
\hline Prunella vulgaris L. & $\mathrm{C}$ & IT & * \\
\hline Salvia aethiopis L. & $\mathrm{H}$ & IT & * \\
\hline Salvia multicaulis Vah1. & $\mathrm{C}$ & IT, KO & * \\
\hline Salvia spinosa L. & $\mathrm{H}$ & IT, KO & * \\
\hline Scutellaria multicaulis Boiss. & $\mathrm{H}$ & IT, KO & \\
\hline Scutellaria nepetifolia Benth. & $\mathrm{H}$ & IT, KO & \\
\hline Scutellaria pinnatifida A. Hamilt. & $\mathrm{H}$ & IT, KO & \\
\hline Stachys acerosa Boiss. & $\mathrm{C}$ & IT, KO & \\
\hline Stachys inflate Benth. & $\mathrm{H}$ & IT, KO & * \\
\hline Stachys lavandulifolia Vah1. & $\mathrm{H}$ & IT, KO, H & * \\
\hline Stachys multicaulis Benth. & $\mathrm{C}$ & IT & * \\
\hline Stachys setifera C. A. Mey. & G & IT, KO & \\
\hline Teucrium orientale L. & $\mathrm{H}$ & IT, KO & * \\
\hline Teucrium polium L. & $\mathrm{H}$ & IT, M, KO & * \\
\hline Thymus daenensis Celak. & $\mathrm{H}$ & IT, Z & \\
\hline Thymus kotschyanus Boiss. \& Hohen & $\mathrm{C}$ & IT, Z & * \\
\hline \multicolumn{3}{|l|}{ LILIACEAE } & * \\
\hline Allium paniculatum L. & G & IT, Z & \\
\hline \multicolumn{4}{|l|}{ LINACEAE } \\
\hline $\begin{array}{c}\text { Linum catharticum L. } \\
\text { LYTHRACEAE }\end{array}$ & \multicolumn{3}{|c|}{ LYTHRACEAE } \\
\hline \multicolumn{3}{|l|}{ MALVACEAE } & * \\
\hline \multicolumn{4}{|l|}{ OLEACEAE } \\
\hline Fraxinus sp. & $\mathrm{H}$ & IT & \\
\hline \multicolumn{4}{|l|}{ ONAGRACEAE } \\
\hline Epilobium hirsutum L. & $\mathrm{H}$ & IT & \\
\hline $\begin{array}{c}\text { Epilobium palustre L. } \\
\text { ORCHIDACEAE }\end{array}$ & $\mathrm{H}$ & IT & \\
\hline Epipactis palustris (L.) Crantz & $\mathrm{H}$ & IT & \\
\hline PAPILONACEAE & & & \\
\hline Astragalus alopecias Pallas & $\mathrm{H}$ & IT & \\
\hline Astragalus caprinus & $\mathrm{H}$ & IT & \\
\hline Astragalus eriopodus Boiss. & $\mathrm{H}$ & IT & \\
\hline Astragalus glaucops Bornm. & $\mathrm{H}$ & IT & \\
\hline
\end{tabular}




\section{Continued}

Astragalus gossypinus Fischer

Astragalus hymenocalyx Boiss.

Astragalus parrowianus Boiss. \& Hausskn.

Astragalus satiger

Cicer anatolicum Alef.

Cicer oxyodon Boiss. \& Hohen

Lotus corniculatus L.

Medicago lupulina L.

Medicago sativa $\mathrm{L}$.

Ononis spinosa $\mathrm{L}$.

Sophora alopecuroides L.

Trifolium pratense $\mathrm{L}$.

Trifolium radicoum Boiss. \& Hohen

PAPAVERACEAE

Papaver rhoeas L.

Papaver dubium L.

Papaver fugax Poir.

PLANTAGINACEAE

Plantago gentianoides Sibth. \& Sm.

Plantago lagopus L.

Plantago lanceolata $\mathrm{L}$.

Plantago major $\mathrm{L}$.

PLUMBAGINACEAE

Acantholimon bromifolium Boiss.

Acantholimon olivieri (Jaub. \& Spach) Boiss.

\section{POACEAE}

Agropyrum elongatiforme Drobov

Agropyrum intermedium P. Beauv.

Agropyrum longe_aristatum Boiss.

Agropyrum pectiniforme Roemer \& Schultes

Agropyrum tauri Boiss. \& Bal.

Agropyrum trichophorum Richter

Agrostis canina L.

Agrostis gigantea Roth.

Arrhenatherum kotschyi Boiss.

Bothriochloa ischaemum (L.) Keng.

Brachypodium sylvaticum P.Beauv.

Bromus danthoniae Trin.

Bromus tectorum L.

Bromus tomentellus Boiss.

Calamagrostis pseudophragmites koel.

Cynodon dactylon (L.) Pers.

Dactylis glomerata L.

Eremopoa persica (Trin.) Roshev

Festuca ovina L.

Festuca rubra L.

Hordeum bulbosum L.

Hordeum violaceum Boiss. \& Huet.
IT, KO, Z, H, IT, KO, H, COS

IT, KO, Z, H

IT, KO, $\mathrm{H}$

IT, KO, $\mathrm{H}$

COS

COS

IT, Z

IT, Z

KO, H, Z, IT

KO, H, Z, IT

KO, H, Z, IT

KO, H, Z, IT

KO, H, Z, IT

KO, H, Z, IT

IT

IT

IT

IT

IT

IT, Z, KO

IT, M, COS

IT

IT

IT, KO, $\mathrm{H}$

IT

IT, KO

IT

IT

Z, IT, H

IT, KO 


\section{Continued}

Leucopoa sclerophylla. Krecz. \& Bobrov

Melica jacquemontii Decne. Ex. Jacquem.

Melica persica Kunth.

Oryzopsis lateralis (Regel) Stapf.

Oryzopsis molinioides (Boiss.)

Phalaris arundinacae L.

Phragamites australis Trin.Ex.Steud.

Poa bulbosa L.

Poa trivialis L.

Stipa barbata Desf.

Taeniatherum crinitum (Schreb) Nevski

Trisetum flavescens (L.) P. Beauv.

POLYGONACEAE

Polygonum bistorta L.

Polygonum luzuloides Jaub. \& Spach

Polygonum paronychioides C. A. Mey, Hohen

Polygonum polycnemoides Jaub. \& Spach.

Rumex chalepensis Miller

Rumex conglomerates Murr.

Rumex crispus L.

PRIMULACEAE

Primula auriculata Lam.

RANUNCULACEAE

Delphi nium. sp.

Ranunculus sericeus Banks \& Soland

ROSACEAE

Alchemilla kurdica Rothm. Ex. Bornm

Alchemilla persica Rothm.

Amaygdalus lycioides Spach

Cerasus microcarpa (C.A.Mey)Boiss.

Cerasus microcarpa (C.A.Mey.)Boiss.

Cotenoaster. sp.

Crataegus pontica C. Koch.

Crataegus pseudoheterophylla Pojark.

Potentilla canescens Besser

Potentilla recta $\mathrm{L}$

Rosa canina L.

Rosa elymaitica Boiss. \& Hausskn.

Rosa orientalis Dupont Ex. Ser.

Rubus anatolicus (Focke.) Focke. Ex. Hausskn.

Rubus saxatilis L.

Sanguisorba minor Scop.

Sibbaldia parviflora Willd.

RUBIACEAE

Asperula glomerata (M. B.) Griseb.

Asperula setosa Jaub. Et. Sp.

Callipeltis cucularis Stev.

Cruciatatau rica Ehrend.
IT, KO

IT, KO

IT

IT, $\mathrm{KO}$

IT, KO

IT, $\mathrm{KO}$

IT, $\mathrm{KO}$

IT, M, Z, H, KO

IT, M

IT

IT

IT

C

H

$\mathrm{H}$

H

$$
\text { IT }
$$

$$
\text { IT }
$$

IT

IT, $\mathrm{H}$

IT, H

IT

IT

IT

IT

IT

IT

IT

IT

IT

IT

IT, KO

IT, $\mathrm{KO}$

\section{IT}

IT

IT, Z

IT, Z 


\section{Continued}

\begin{tabular}{|c|c|c|c|}
\hline \\
\hline Galium aparine L. & $\mathrm{T}$ & IT, Z & $*$ \\
\hline Galium mite Boiss. \& Hoh. & $\mathrm{C}$ & IT & \\
\hline Galium setaceum Lam. & $\mathrm{T}$ & IT, KO, Z & \\
\hline Galium verum L. & $\mathrm{C}$ & IT & $*$ \\
\hline Rubia tinctorum L. & $\mathrm{H}$ & IT & \\
\hline \multicolumn{4}{|l|}{ SALICACEAE } \\
\hline Salix acmophylla Boiss. & $\mathrm{P}$ & IT & \\
\hline Salix. sp. & $\mathrm{P}$ & IT & \\
\hline \multicolumn{4}{|l|}{ SCROPHULARIACEAE } \\
\hline Linaria grandiflora Desf. & $\mathrm{H}$ & IT & \\
\hline Scrophularia azerbaijanica Grau. & $\mathrm{H}$ & IT, KO & \\
\hline Scrophularia nervosa Benth. & $\mathrm{H}$ & IT, KO & \\
\hline Scrophularia variegata M. B. & $\mathrm{H}$ & IT, KO & \\
\hline Verbascum agrimoniifolium & $\mathrm{C}$ & IT & \\
\hline Verbascum nudicaule (wydl.)Takht. & $\mathrm{C}$ & IT & \\
\hline Verbascum songaricum Schrenk Ex. Fisch. \& C. A. Mey. & $\mathrm{C}$ & IT & $*$ \\
\hline Verbascum speciosum Schrad. & $\mathrm{H}$ & IT, KO & * \\
\hline Veronica anagallisaquatica $\mathrm{L}$. & $\mathrm{H}$ & IT & * \\
\hline \multicolumn{4}{|l|}{ THYMELAEACEAE } \\
\hline Dendrostelleralessertii Van Tiegh. & $\mathrm{C}$ & IT & \\
\hline \multicolumn{4}{|l|}{ UMBELLIFERAE } \\
\hline Bupleurum Exaltatum M. B. & $\mathrm{H}$ & IT & \\
\hline Bupleurum gerardii All. & $\mathrm{H}$ & IT & \\
\hline Echinophora platyloba Dc. & $\mathrm{H}$ & IT & \\
\hline Eryngium billardieri F. Delaroche & $\mathrm{H}$ & H, IT & \\
\hline Eryngium pyramidale Boiss. \& Housskn. & $\mathrm{H}$ & H, IT & \\
\hline Ferula orientalis L. & $\mathrm{H}$ & IT & \\
\hline Grammosciadium platycarpum Boiss. \& Hausskn. & $\mathrm{T}$ & IT & \\
\hline Leutea gracillima M. Pimen. & $\mathrm{H}$ & IT & \\
\hline Peucedanum sp. & $\mathrm{H}$ & IT & \\
\hline Pimpinella affinis Ledeb. & $\mathrm{H}$ & IT & \\
\hline Pimpinella aurea Dc. & $\mathrm{H}$ & IT & \\
\hline Pimpinella tragium Vill. & $\mathrm{H}$ & IT & \\
\hline Prangosu loptera Dc. & $\mathrm{H}$ & IT & \\
\hline Rhabdosciadium aucheri Boiss. & $\mathrm{H}$ & IT & \\
\hline Rhabdosciadium petiolare Boiss. \& Hausskn. & $\mathrm{H}$ & IT & \\
\hline Siumsis aroideum Dc. & $\mathrm{H}$ & IT & \\
\hline \multicolumn{4}{|l|}{ URTICACEAE } \\
\hline Urtica dioica $\mathrm{L}$. & $\mathrm{H}$ & IT, KO & * \\
\hline \multicolumn{4}{|l|}{ VALERIANACEAE } \\
\hline Valerianasis ymbriifolia Vah1. & $\mathrm{H}$ & IT, KO & \\
\hline
\end{tabular}

The low percentage of Chamaephyte, Geophytes and Phanerophyte shows that they are not adapted to existence climate and edaphically situations. Each plant species has its special ecological area with a known tolerance to life conditions of area. Therefore, the geographical distribution of plant species depending on life conditions of area and adaptation of plants to area [16].

Astragalus diversity with its 8 species in this area which is mountainous shows that Astragalus has adapted to the mountainous conditions. 


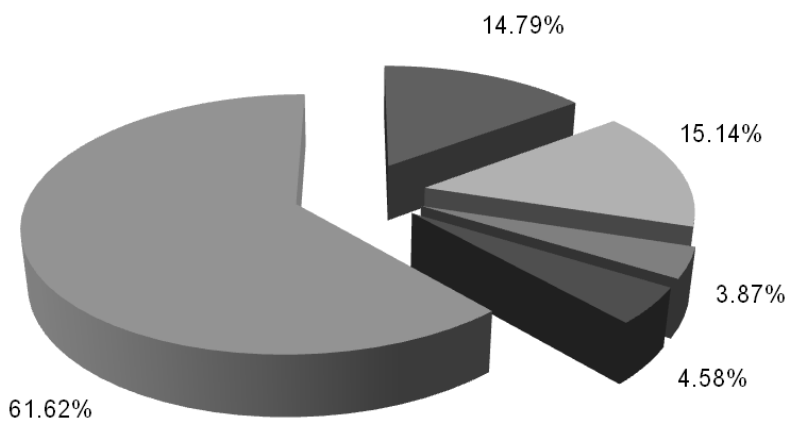

Figure 3. The pie chart of life form of species Hamedan Alvand region.

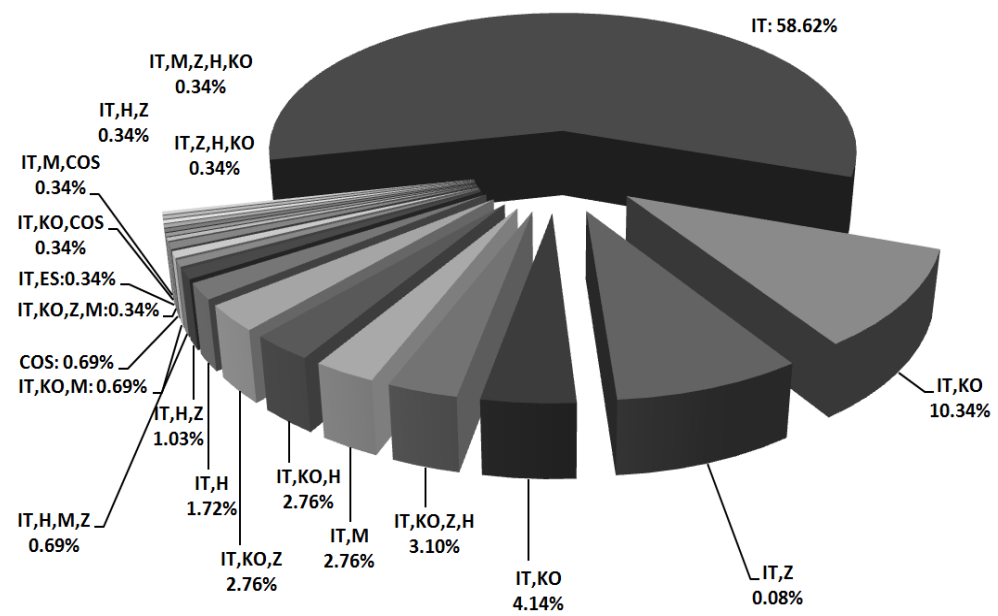

Figure 4. The pie chart of percentage of phytochoria of species Hamedan Alvand region.

The Chorotype distribution of plants reflects the climate conditions. Considering to this fact that $59 \%$ plant species in a region are IT elements, so this region belong to IT. IT (the Irano-Turanian region) is characterized by low rainfall and a long dry season.

The existence of Compositae family with large diversity is the result of destruction in this region. It is experience understood that the increasing of the number of some plant families including Asteraceae accompanied with destruction in area, following studies support the mentioned fact [15] [18].

About 71 medicinal species were determined in this area that was shown in Table 1. Presence of important medicinal plants is indicated Potential of region as pool of medicinal plants.

Some of most important species area as follows: Thymus kotschyamus, Ziziphora clinopodioides, Stachys inflata, Stachys lavandulifolia. The presence of high biodiversity, visual and historical regions as: Ganjnameh, eye-catching landscape of Alvand top, Takhteh-Nader, MeydanMishan, Kivarestan, have encouraged research, educational and tourist activities in the region.

The rapid development of tourism industry, Tele Cabin in recent years has had a significant effect on the vegetation and landscape. Ganjnameh has a long history of human occupation with ancient civilizations well reflected in the archaeological records.

Existence of ruderal plant such as Cardariacraba in this region showed anthropogenic impacts. As human population levels and resource requirements continue to grow, function of ecosystem (net primary productivity) and species richness distributions are likely to be increasingly affected by anthropogenic land-use [17] [19] [20].

The research in the Alvand Hamedan region has revealed the importance of this region in terms of plant biodiversity, and particularly of touristic characteristics, which urgently require further management and conservation activities. 


\section{Acknowledgements}

This work was funded by a grant from Bu Ali Sina university and Shahid Chamran University of Ahvaz Research Council Grant No: Grant No: (p.2.2186, 1392.10.8). The authors declare that there is no conflict of interest.

\section{References}

[1] Eftekhari, T. and Ramezani, M. (2004) Introduction to Plant Biodiversity in Iran. In: Pushpangadan, P., Nair, K.N. and Ahmad, M.R., Eds., Biodiversity and Medicinal Plant Wealth of South Asian Countries, National Botanical Research Institute, Lucknow, 39-40.

[2] Hundera, K., Bekele, T. and Kelbessa, E. (2007) Floristics and Phytogeographic Synopsis of a Dry Afromontane Coniferous Forest in the Bale Mountains, Ethiopia: Implications to Biodiversity Conservation. Ethiopian Journal of Science, 30, 1-12.

[3] Mengistu, K. (2002) Tropical Secondry Forest Management in Africa: Reality and Perspectives: Ethiopia Country Paper. FAO Document Repository, 13, 43-58.

[4] Yavari, A., Shahgolzari, S.M. and Atri, M. (2010) Application of Floristic Marker in Eco-Phytosociology Method for Diagnosing Existing Intraspecific Diversity in Plants: A Case Study of Astragalusglaucops. International Journal of Agriculture and Biology, 12, 887-890.

[5] Atri, M. (1996) A Presentation of Some Aspects of the Application of Neosigmatiste Method in Pedology, Systematics and Chorology. Iranian Journal of Biology, 2, 57-63.

[6] Rechinger, K.H. (2005) Flora Iranica. Vol. 1-176, Akademishe Druck University, Graz.

[7] Akhani, H. (2005) The Illustrated Flora of Golestan National Park Iran. Vol. 1. University of Tehran Press, Tehran.

[8] Akhani, H. (2006) Flora Iranica: Facts and Figures and a List of Publications by K. H. Rechinger on Iran and Adjacent areas. Rostaniha, 7, 19-61.

[9] Akhani, H. (2007) Diversity, Biogeography and Photosynthetic Pathways of Argusia and Heliotropium (Boraginaceae) in South-West Asia with an Analysis of Phytogeographical Units. Botanical Journal of the Linnean Society, 155, 401-425.

[10] Ghahraman, A. (1979-1998) Colorful Flora of Iran. The Research Institute of Forest and Pastures, Tehran. Implication to Biodiversity Conservation. SINET: Ethiopian Journal of Science, 30, 1-12.

[11] Mobayen, S. (1996) Flora of Iran 1980-1996. Vol. 1-4, Tehran University Press, Tehran.

[12] Assadi, M., Massoumi, A.A., Khatamsaz, M. and Mozaffarian, V. (2008) Flora of Iran 1988-2008. Vol. 1-60, Research Institute of Forest Publication, Tehran. (in Persian)

[13] Zohary, M. (1973) Geobotanical Foundations of the Middle East. Vol. 2, Gustav Fisher Verlag, Stuttgart.

[14] Raunkier, C. (1934) Life Forms of Plants. Oxford University Press, Oxford.

[15] Archibold, O.W. (1995) Mediterranean Ecosystems: Ecology of World Vegetation. Chapman Hall, London, $131-164$.

[16] Asri, Y. (2003) Plant Diversity in Touran Biosphere Reservoir. Vol. 305, Research Institute of Forests and Rangelands, Tehran, 306.

[17] Kolahi, M. and Atri, M. (2014) The Effect of Ecological Factors on Vegetation in Hamedan Alvand Region (Iran). International Journal of Farming and Allied Sciences, 3, 489-496.

[18] Vakili-Shahrebabaki, M., Atri, M. and Assadi, M. (2001) Floristic Study of Meymand Shahrehbabak and Identification Biological Forms and Chorotype of Area Plants. Iranian Journal Pajouheshnd Sazandegi, 52, 75-81.

[19] Al Khulaidi, A.W., Miller, A.G. and Furley, P. (2007) Environmental and Human Determinates of Vegetation Distribution in the Hadhramaut Region, Republic of Yemen.

[20] Williams, J., Eric, W., Seabloom, A., Slayback, D., Stoms, D.M. and Viersm, J.H. (2005) Anthropogenic Impacts upon Plant Species Richness and Net Primary Productivity in California. Ecology Letters, 8, 127-137. http://dx.doi.org/10.1111/j.1461-0248.2004.00706.x 